\title{
Blind Identification of Photorealistic Computer Graphics Based on Fractal Dimensions
}

\author{
Yingda Lv, XuanJing Shen, Guofu Wan, HaiPeng Chen \\ College of Computer Science and Technology, JiLin University, Changchun, Jilin Province, China \\ $\{$ xjshen \& chenhp\}@jlu.edu.cn, \{gfwan10\&lvyd10\}@mails.jlu.edu.cn
}

\begin{abstract}
The perceptual differences between photographic images and CG primarily exist in the colour and the surface texture. In this study a blind identification algorithm was proposed to identify $\mathrm{CG}$, which is based on fractal dimensions. Firstly, change the image's colour space from RGB to HSV and extract fractal dimensions on HSV colour space; Secondly, obtain fractal dimensions from image's gradient image; Thirdly, calculate fractal dimensions of PEM and the global fractal dimension. The experimental results showed its effectiveness, and the correct detection rate of CG is $96 \%$.
\end{abstract}

Index Terms - Photorealistic Computer Graphic, Fractal Dimension, Colour Space, Gradient image

\section{Introduction}

With the developments of information technology, the tampering technology of digital images has been improved rapidly. People can easily draw the pictures by computer software so that it is difficult to identify them.

There are many methods to identify photographic images and photorealistic computer graphics (CG). According to [1, 2], although $\mathrm{CG}$ are difficult to be distinguished from photographic images by human eyes, we can extract some features to distinguish them. The features are extracted by calculating statistical moments of image's wavelet sub-bands and its linear prediction error, and identifying photographic images and CG by adaptive classifier. According to [3], some features are proposed, which contain photographic image statistics such as image power spectrum, the wavelet transform and the local image patches. According to [4,5], there is a vital difference between photographic images and $\mathrm{CG}$, the proposed method is based on the differences which are formed in the image generation processes by using devices and the method is independent of the image content. Pattern noise can be used to identify photographic images and CG. According to [6], traces of demosaicking and chromatic aberration are used to differentiate $\mathrm{CG}$ from photographic images and the experimental results are encouraging.

In this paper, we find that the vital differences between photographic images and CG primarily exist in the colour and the surface texture of images, so we extract features from this viewpoint. The feature vector is made up of these features, and we use SVM to classify images. The experimental results show the effectiveness of this method.

\section{Framework of the Method}

There are large differences between photographic images and $\mathrm{CG}$ in the image generation processes, so we can identify categories of these images according to the differences, which exist in the colour and the surface texture. Refs. [7, 8] maintain that colour space information can be used to classify photographic images and $\mathrm{CG}$. The colours of $\mathrm{CG}$ always appear inauthentic and $\mathrm{CG}$ are smoother than photographic images on object surface. We can use fractal dimensions described in [9-12] to capture the differences, and the features can form feature vector, which can be used to identify images.

Firstly, the image's colour space is transformed from RGB to HSV, and the fractal dimension is calculated on HSV colour space; secondly, calculate the fractal dimensions of gradient images; thirdly, calculate the fractal dimensions of prediction error matrix (PEM); finally, compute the global fractal dimension. The detail framework of the method is as shown in Fig. 1.

\section{Features Extraction}

A. Global fractal dimension

Fractal is self-similar phenomena, images, and physical processes. That is to say local features are similar to the overall characteristics. We use "dimension" to describe the irregularity and the complexity of phenomena, images, and physical processes.

In this study, the cubic boxes are calculated to extract fractal dimensions. The process is as follows:

1) Images are divided into many blocks with the size of $K \times K$. The image blocks are considered as a three-dimensional space, $(x, y)$ stands for two-dimensional flat coordinate, and the third dimension stands for image's pixel value.

2) The two-dimensional flat $(x, y)$ is divided into many grids with the size of $l \times l$, where $l$ is an integer and $1 \leq l \leq K / 2$, let $r=l / K$.

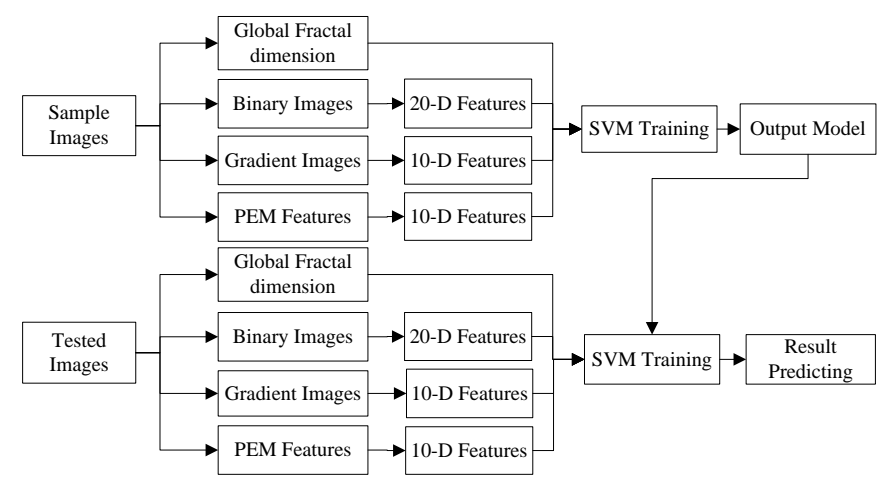

Fig.1 The framework of the method 
3) In each grid, there are many cubic boxes with the size of $l \times l \times l$, the variable $\max _{k}$ denotes maximum of the $k$-th grid's pixel values, and $\min I_{k}$ denotes minimum of the $k$-th grid's pixel values.

4) Let $n_{r}(k)$ denote the number of cubic boxes, the calculating expression is

$$
n_{r}(k)=\left(\max I_{k}-\min I_{k}+1\right) / l .
$$

Let $N_{r}$ denote the sum of all the grids' $n_{r}(k)$, so we can get

$$
N_{r}=\sum_{k} n_{r}(k)
$$

5) Use the least-squares method to fit $\lg N_{r}-\lg (1 / r)$, then fractal dimension is the slope of the fitting curve.

Compared with typical methods, we did not use fixed size of cubic boxes any longer in this approach. However, cuboids with a variable length are used into wrapped surfaces, this approach makes cubic boxes' wrapping more closely, and it can improve the accuracy of the fractal dimension.

\section{B. Fractal dimension of HSV colour space}

The differences between photographic images and CG exist in the colour, that is to say the colours of CG always appear inveracious, so the differences in the colour space are important factors to identify photographic images and CG. In this paper, fractal dimensions are extracted on HSV colour space. The process is as follows:

1) Transform image's colour space from RGB to HSV.

2) Get binary images on hue channel and saturation channel by (3).

$$
I_{k}(m, n)= \begin{cases}1, & (k-1) \times \frac{1}{N} \leq I(m, n) \leq k \times \frac{1}{N} \\ 0, & \text { else }\end{cases}
$$

Where $I(m, n)$ denotes the hue channel or the saturation channel of the HSV colour space, $I_{k}(m, n)$ denotes the $k$-th binary image, $1 \leq k \leq N$. Let $N$ denote the quantity of binary images.

3) Calculate fractal dimensions of the binary images.

Fig.2 is an example to decompose the hue channel (Fig.2 (a)) into 5 binary images (Fig.2(b)-(f)). The fractal dimensions of Fig.2 (b)-(f) are 2.5217, 2.5507, 2.7182, 2.0940 and 2.0591 correspondingly.

\section{Fractal dimension of PEM}

Sources of photographic images are vastly different, and the natural scenery is varied, so local features of photographic images are not the same. CG is just as well. In order to improve the method's accuracy and adaptability, the fractal dimensions are firstly extracted from PEM, which can better measure the surface texture of images.

For each element $x$, the predicted value $\bar{x}$ of element $x$ is

$$
\bar{x}=\operatorname{sign}(x) \cdot\{|a|+|b|-|c|\}
$$

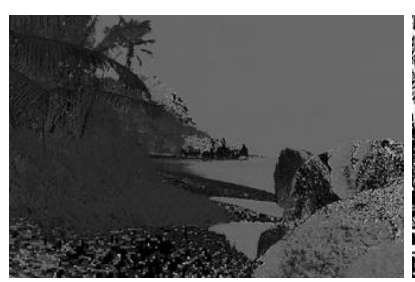

(a)

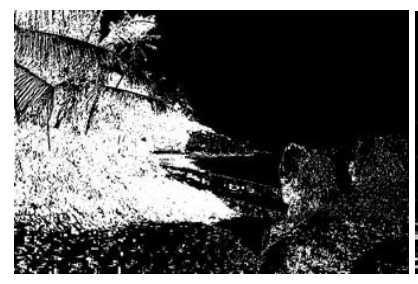

(c)

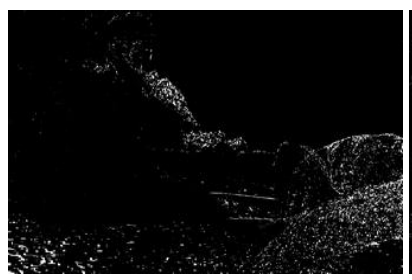

(e)

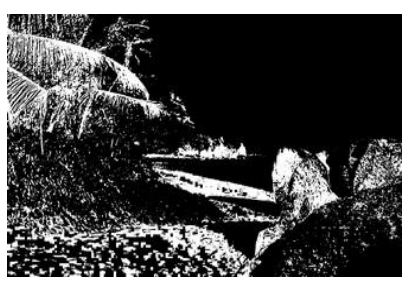

(b)

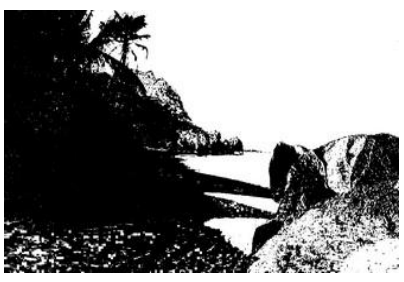

(d)

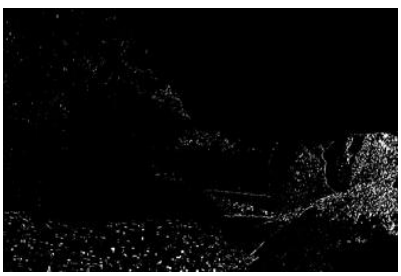

(f)
Fig.2 Decomposing hue channel into 5 binary images. (a) Hue channel; (b) Values of pixels are between 0 and 0.2; (c) Values of pixels are between 0.2 and 0.4 ; (d) Values of pixels are between 0.4 and 0.6 ; (e) Values of pixels are between 0.6 and 0.8 ; (f) Values of pixels care between 0.8 and 1 .

Where $a, b$ and $c$ are the elements in its prediction neighbourhood.

The predicted error value $\Delta x$ is

$$
\Delta x=x-\bar{x}=x-\operatorname{sign}(x) \cdot\{|a|+|b|-|c|\}
$$

PEM can be gotten by calculating every element's predicted error value in the matrix.

PEM is used to calculate the fractal dimensions, which can capture the differences between photographic images and CG. PEM can describe correlation between image's pixel and its neighbourhood pixels, the volatility and complexity of an image can be gotten by calculating fractal dimensions of PEM.

\section{Fractal dimension of gradient image}

Photographic images are different from CG in surface texture of images. In [4], $\mathrm{Ng}$ thought that $\mathrm{CG}$ are smoother photographic images, and used global fractal dimension to measure the smooth degree. But the fractal dimension is only global fractal dimension, and $\mathrm{Ng}$ did not consider local information. In this paper, we use multi-fractal dimensions to capture the image's inner structure and texture differences.

Let $I$ denote an image, $\operatorname{grad}_{I}$ denote gradient image of image I. $\operatorname{grad}_{I}$ can be obtained by (6).

$$
\operatorname{grad}_{I}=\sqrt{\frac{\partial I^{2}}{\partial x}+\frac{\partial I^{2}}{\partial y}}
$$


Let $B_{i}$ be the cubes of the $\delta$-coordinate mesh on the image and $i$ be the index of cube. The measurement $\mu\left(B_{i}\right)$ is the ratio of the sum of gradient intensity in cube $B_{i}$ to the sum of that in the whole image, which is a probability mass function. $\mu\left(B_{i}\right)$ can be calculated by (7)

$$
\mu\left(B_{i}\right)=\frac{\sum_{B_{i}} \operatorname{grad}_{I}(m, n)}{\sum_{I} \operatorname{grad}_{I}(m, n)}
$$

Define the sums over the $\delta$-mesh cubes, which is the $q$-th moment.

$$
S_{\delta}(q)=\sum_{i} \mu\left(B_{i}\right)^{q}
$$

For a constant $\delta, S_{\delta}(q)$ decreases as $q$ increases and $S_{\delta}(q)$ obeys power laws as $\delta \rightarrow 0$, which can be expressed in detail as follows:

$$
\tau(q)=\lim _{\delta \rightarrow 0} \frac{\log S_{\delta}(q)}{-\log \delta}
$$
follows:

Then multi-fractal dimensions can be expressed as

$$
D_{q}=\frac{\tau(q)}{q-1}
$$

Fig. 3 shows the image of multi-fractal dimensions. From fig. 3 we find that when $q$ is smaller than -10 or larger than 0 , $D_{q}$ changes slowly, so we focus on some important $q$ values, and if these $q$ values change a little, $D_{q}$ changes obviously. So we give the values of $q$ as $-10,-8,-7,-6,-5,-4,-3,-2,-1$ and 0 . Both gradient image and PEM need calculate multi-fractal dimensions, these features form 20 dimensions feature vector.

\section{Experiments Results and Analysis}

\section{A. Classifier}

High dimensions features are often linearly inseparable, so SVM classifier is selected in this method. The kernel function is Radial basis function, and LibSVM3.0 is selected as implementation tools. All image data uses cross validation to find the optimal parameter set.

\section{B. Image database}

In our experiment, we select 1600 JPEG images as original image set, 800 images of them are photographic images, and they are downloaded from Columbia University, the other images are downloaded from the following websites:

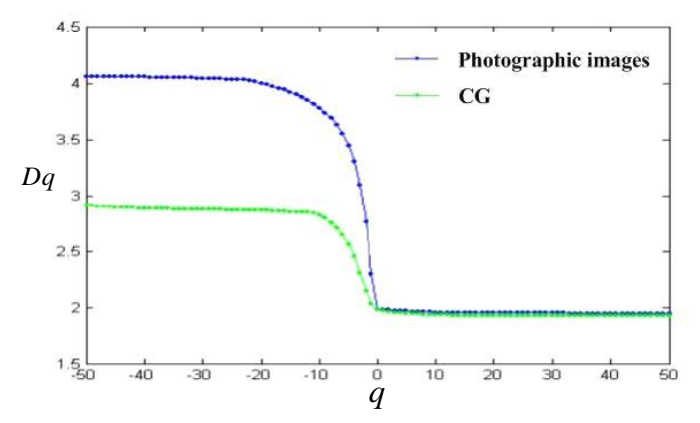

Fig.3 The image of gradient image's multi-fractal dimension

www.3dshop.com, www. Raph.com. The contents are diverse, including buildings, persons, sceneries, etc.

\section{Experiment Process}

Before training, six-fold cross validation on the whole images data was used to find optimal parameter set, that is to say, in the process of finding optimal parameter set, for any parameter set, the entire training data was randomly divided into six parts, and then five parts of them was used to train and the rest to test, repeat this process till all the samples are already tested. At last, the prediction accuracy can be gotten for all the data, so optimal parameter set can be judged from different results by using different parameter sets. In our experiment, 400 photographic images and $400 \mathrm{CG}$ are selected as training samples, and the rest are tested samples. The detail experiment process is as follows:

a) Read an input image and get its category (Photographic Image $=1$ and $C G=-1$ ).

b) Extract image's features.

c) Repeat a) to b) till all of input images' features are extracted completely.

d) Use easy.py to do cross validation to find the optimal parameter in the training mode.

e) Establish SVM model using image's features and the optimal parameter.

f) Identify testing images using SVM model.

\section{Results and analysis}

The features of the experiment contain global fractal dimension, fractal dimension of HSV colour space, fractal dimension of PEM, fractal dimension of gradient image. In the process of extracting fractal dimension of HSV colour space, 10 binary images are calculated on both hue channel and saturation channel, so we can get 20 fractal dimensions features. The quantity of fractal dimensions features for both PEM and gradient image is equal to the quantity of $q$ values, this paper give the values of $q$ are $-10,-8,-7,-6,-5,-4,-3,-2$, -1 and 0 , so we can also get 20 fractal dimension features.

From fig.3, we can conclude that there are large differences in multi-fractal dimensions features, especially when $q$ equals $-10,-8,-7,-6,-5,-4,-3,-2,-1$ and 0 , the difference is obvious. If only use fractal dimensions of PEM as features, classifying accuracy rate achieves $77.2 \%$. 
Table I is classifying accuracy rate comparison table between our method and [1], TP stands for detecting accuracy of CG and TN stands for detecting accuracy of photographic images. The average accuracy is the arithmetic mean of TP and TN. This method has an ideal detecting accuracy. The receiver operating characteristics (ROC) curve is shown in Fig.4.

TABLE I The Detecting Accuracy of Photographic Images and CG

\begin{tabular}{|c|c|c|c|}
\hline & $\mathrm{TN} / \%$ & $\mathrm{TN} / \%$ & Accuracy $/ \%$ \\
\hline Ref. $[1]$ & $94.22 \%$ & $91.18 \%$ & $92.7 \%$ \\
\hline Our method & $96 \%$ & $88 \%$ & $92 \%$ \\
\hline
\end{tabular}

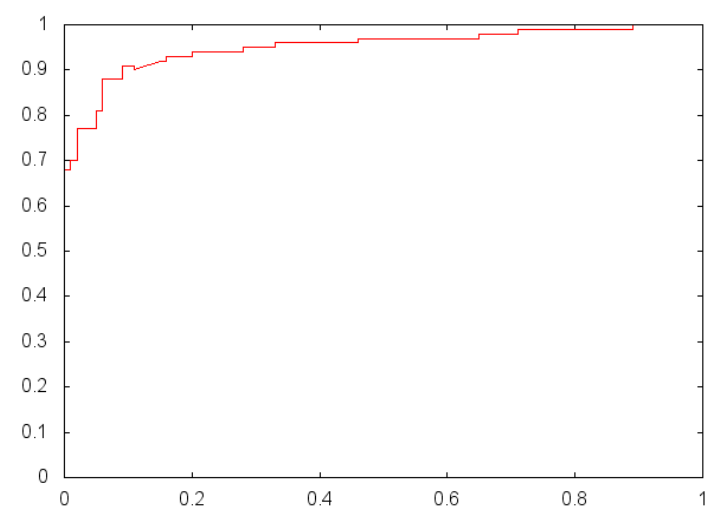

Fig.4 ROC Curve(AUC=0.9516)

From the experimental results we can find that so many features can be used to identify CG. The fractal dimension can measure the complexity of image surface texture. The features on PEM can evaluate the correlation among image pixels. The features on HSV colour space can estimate distribution of image's colour space information. By using the features above, we get improve detection accuracy for identification CG.

\section{Conclusions}

This paper proposes an effective method to identify CG. The feature vector can be formed by calculating fractal dimensions of HSV colour space, fractal dimensions of PEM and gradient image, and global fractal dimension. The experimental results show that this method achieves good performance.
The main contributions of this paper are as follows:

(1) We have proposed and analysed the difference in surface texture between photographic images and $\mathrm{CG}$, and to have given a way to measure it.

(2) We have analysed the difference in pixel correlation between photographic images and CG. Based PEM, we have proposed a method to measure this difference using fractal dimension.

\section{Acknowledgment}

Our research is supported by National Natural Science Grant (No.61305046), Natural Science Grant of Jilin Province (NO.201115025), and Graduate Innovation Fund of Jilin University (NO. 20111063).

\section{References}

[1] Siwei Lyu, Hany Farid. "How Realistic is photorealistic". IEEE Trans Signal Process, 53(2), 2005, pp. 845-850.

[2] Farid H, Lyu S. "Higher-order wavelet statistics and their application to digital forensics". IEEE Workshop on Statistical Analysis in Computer Vision, 2003.

[3] Tian-Tsong Ng, Shih-Fu Chang. "Classifying photographic and photorealistic computer graphic images using natural image statistics". Technical Report, Columbia University, 2004,10.

[4] Nitin Khanna, George T.-C. Chiu, Jan P. Allebach, et al. "Forensic techniques for classifying scanner, computer generated and digital camera images". ICASSP, Las Necada, U.S.A. 2008, pp. 1653-1656.

[5] Weihai Li, Nenghai Yu, Yuan Yuan. "Identifying Camera and Processing from Cropped JPEG Photos via Tensor Analysis”. 2010 IEEE, Istanbul, Turkey, 2010, pp.3889-3895.

[6] A. E. Dirik, S. Bayram, H. T. Sencar, N. Memon. "New Features to Identify Computer Generated Images". IEEE International Conference on Image Processing, 2007, pp.433-436.

[7] Amanatides J. Realism in computer graphics: a survey. IEEE Comp Graph Appl, 1987, pp. 44-56.

[8] Chen W, Shi Y, Xuan G. Identifying computer graphics using HSV colour model and statistical moments of characteristic functions. In: Multimedia and Expo, 2007 IEEE International Conference, 2007. 1123-1126.

[9] Dongmei Chen, Jianhua Li, Shilin Wang. "Identifying Computer Generated and Digital Camera Images Using Fractional Lower Order Moments". The 4th IEEE Conference on Industrial Electronics and Applications, Xi'an, China, 2009, pp. 230-235.

[10] MIN SHAO, CHRYSOSTOMOS L. "Signal processing with fractional lower order moments stable processes and their applications". PROCEEDINGS OF THE IEEE, 81 (7), 1993, pp. 986 -1010.

[11] PU YiFei, WANG WeiXing, ZHOU JiLiu, et al. "Fractional differential approach to detecting textural features of digital image and its fractional differential filter implementation". Sci China Ser F, 51(9), 2008, pp. 1319-1339.

[12] Tian-Tsong Ng, Shih-Fu Chang, Mao-Pei Tsui. "Physics-motivated features for distinguishing photographic images and computer graphics". in ACM Multimedia, Singapore, 2005,11 